\title{
Allergen Immunotherapy for Allergic Rhinitis
}

\author{
Kimihiro Okubo and Minoru Gotoh \\ Department of Head \& Neck and Sensory Organ Science, Graduate School of Medicine, Nippon Medical School
}

\begin{abstract}
Subcutaneous immunotherapy (SCIT) and sublingual immunotherapy (SLIT) are the 2 forms of desensitization for allergic diseases which are used internationally. Despite the lack of sufficient evidence of the mechanism, SCIT has been used in Japan. When SCIT is performed appropriately, it is effective for allergic rhinitis, as supported by high-level evidence, including the results of meta-analysis. However, its use in Japan has not become widespread due to various problems, including the risk of anaphylaxis. Therefore, attention is being focused on SLIT because it is easier to perform but may be similarly effective and has been extensively discussed and evaluated internationally. Comparison of SCIT and SLIT can only be done with some allergen, which has also been discussed extenesively. Many patients and physicians would welcome the use of SLIT in Japan, and its first use against pollinosis due to Japanese cedar pollen is planned for 2014 .
\end{abstract}

(J Nippon Med Sch 2010; 77: 285-289)

Key words: subcutaneous immunotherapy, sublingual immunotherapy, regulatory $\mathrm{T}$ cell, pollinosis

\section{Introduction}

Allergic rhinitis (AR) is a typical type I allergic disease in the classical classification of Gell and Coombs and is recognized as a chronic lifestyle disease by the Japanese Ministry of Health and Welfare. It reduces quality of life (QOL) even if it is unlikely to cause death. The recent increase in prevalence has been marked for atopic dermatitis and asthma as well as for AR. It is not possible to achieve advances in the treatment of other diseases if AR (a simple type I allergy) cannot be controlled.

Because AR is caused by specific allergens, immunotherapy is an important form of treatment. However, immunotherapy is not widely used in
Japan because of the risk of anaphylaxis and the high cost. Subcutaneous immunotherapy (SCIT) was first performed by Noon in 1911. Therefore, this treatment has been used for nearly a century, and much evidence has been accumulated. The latest evidence about immunotherapy is considered in this editorial.

\section{SCIT}

SCIT is a treatment for allergen-specific allergic diseases (Fig. 1) which involves the subcutaneous injection of gradually increasing doses of the allergen, with the aim of decreasing the patient's responsiveness. SCIT is mentioned in the "Practical Guideline for Management of Allergic Rhinitis in

Correspondence to Kimihiro Okubo, Department of Otolaryngology, Nippon Medical School, 1-1-5 Sendagi, Bunkyoku, Tokyo 113-8602, Japan

E-mail: ent-kimi@nmsc.ac.jp

Journal Website (http://www.nms.ac.jp/jnms/) 


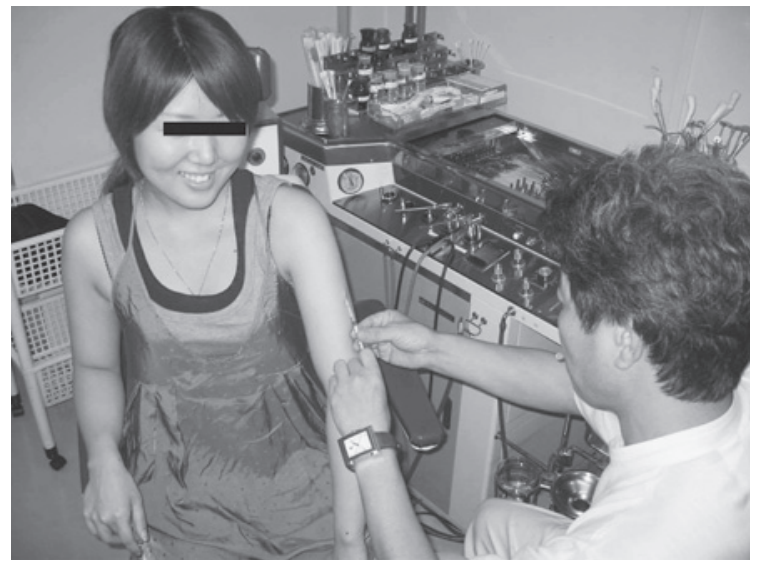

Fig. 1 SCIT.

Japan 2009"2, and in "Allergic rhinitis and its impact on asthma (ARIA)"3, which is an international guideline. The following features of SCIT were described in the immunotherapy opinion book published by the World Health Organization.

1. SCIT can be used for treatment of AR alone or with other methods.

2. SCIT is also effective for allergic conjunctivitis and allergic asthma.

3. SCIT should be performed by an allergy specialist.

4. Only standard allergens should be used.

5. The amount of allergen is increased gradually from a low dose until the maintenance dose is reached.

6. The maintenance dose is usually 5 to $20 \mu \mathrm{g}$ of allergen per injection.

7. There is a risk of anaphylaxis, and appropriate precautions are necessary in case of emergency.

8. Treatment is generally continued for 3 to 5 years, although the optimum time is uncertain.

\section{Efficacy, Mechanism, and Problems with SCIT}

The subjective efficacy rate of SCIT for AR due to house dust (HD) mite is $80 \%$, and the efficacy rate in the United States for the treatment of AR due to ragweed has been reported to be $90 \%$ or more ${ }^{4}$. In Japan, an efficacy rate of about $70 \%$ has been reported for pollinosis caused by Japanese cedar (JC) pollen, even allowing for seasonal variations in the pollen count. Persistence of the effect after the completion of treatment has been confirmed

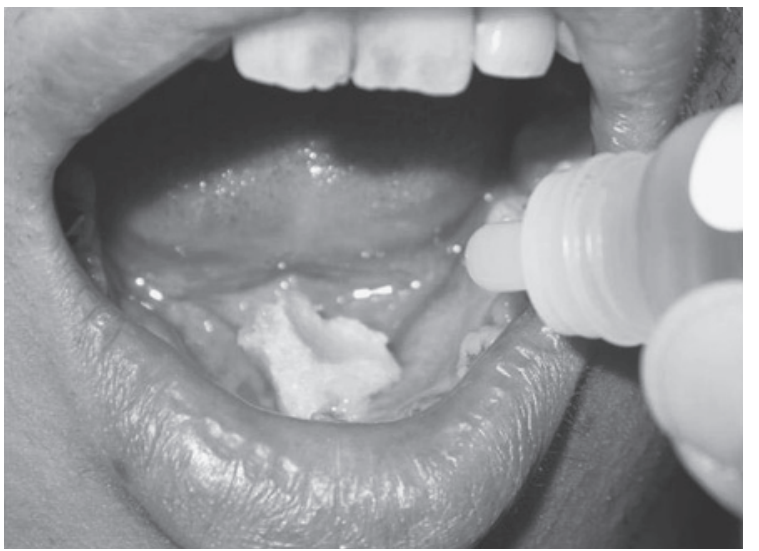

Fig. 2 SLIT.

internationally ${ }^{5}$.

An efficacy rate of $80 \%$ to $90 \%$ has been reported for atopic asthma with moderate symptoms ${ }^{6,7}$. Effectiveness for occupational allergy has also been reported.

SCIT is not considered effective for skin diseases, and it is not found in the guidelines for standard therapy despite some reports of its effectiveness in foreign countries.

There is considerable evidence for the efficacy of SCIT for bronchial asthma and AR in children". Moreover, if SCIT controls pollinosis in infants, asthma can be prevented ${ }^{9}$. However, the frequency of an exaggerated immune response (anaphylaxis) to SCIT is greater in children than in adults.

Although the mechanism of action of SCIT has not been clarified, SCIT might depend on the suppression of $\mathrm{IgE}$ production ${ }^{10,11}$, production of blocking antibodies ${ }^{10-12}$, a decrease of mast cells in nasal mucosa ${ }^{13}$, a decrease of cytokine production (down regulation of interleukin $5 \mathrm{mRNA})^{14}$, and an increase of Th1 cytokines ${ }^{15}$.

Thus, induction of tolerance or anergy, alteration of the Th1/Th2 balance, and regulation of $\mathrm{T}$ cells ${ }^{16,17}$ may be involved in the immunologic mechanism of SCIT. However, the failure to completely clarify these mechanisms helps prevents the widespread use of SCIT.

The main problem with SCIT is the risk of systemic side effects. We have reported 17 cases of side effects in 1,642 patients with AR who underwent SCIT at the Department of Otolaryngology of Nippon Medical School from 1979 
Changing of total symptom score in pollen season 2005 by SLIT

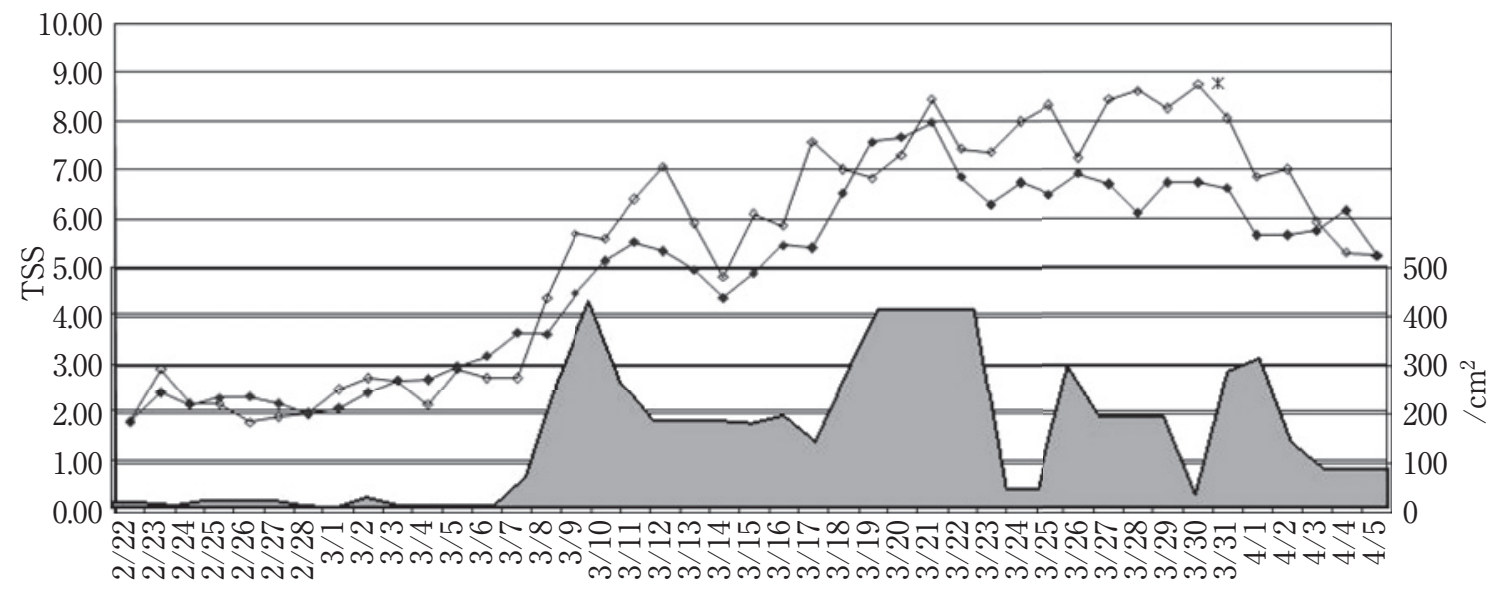

$\diamond$ placebo $\rightarrow-$ active

Start of pollen dispersing on February 22th

10,625 pollens $/ \mathrm{cm}^{2} /$ season by Darham sampler

Fig. 3 JC pollen was present at high levels in 2005 (placebo-controlled study). The solid area shows the changes in the pollen count. Open squares represent the total symptom score (TSS) in the placebo group, and solid squares represent the TSS in the group given JC allergen.

through 1990. The clinical records in 5 of these cases were found to be incomplete. If these cases are excluded, the rates of eruptions, respiratory symptoms, and circulatory symptoms were $1 \%$ or less in the total patients base, which was $1 / 10$ th of the frequency in the total injection base ${ }^{18}$.

\section{SCIT and Sublingual Immunotherapy}

Sublingual immunotherapy (SLIT) has been reported as an alternative form of immunotherapy. SLIT can affect numerous lymph nodes (oral lymph nodes, Waldeyer ring, and cervical nodes) and is more useful than SCIT because of its simplicity and safety (Fig. 2). A clinical trial of SLIT for JC pollinosis was started in our department because its efficacy has often been reported ${ }^{19}$.

Two studies have compared the effects by means the double-dummy method, although few comparisons of SCIT and SLIT have assessed efficacy, cost, and other factors. Thirty-six patients with AR and asthma due to house dust mites were evaluated in the first randomized study in $1999^{20}$. Symptoms of asthma improved only with SCIT, but symptoms of rhinitis decreased with either SCIT or
SLIT $^{20}$. However, the asthma symptom score before immunotherapy was higher in the SCIT group than that in the SLIT group. In another study, 58 patients with silver birch pollinosis were randomly assigned to receive $\operatorname{SCIT}(n=19)$, SLIT $(n=14)$, or a placebo $(n=15)$. This study was small, but improvement in symptoms was significantly greater with SCIT or SLIT than with placebo but did not differ between the two immunotherapy methods; however, a difference might have been found if more cases were included $^{21}$. Randomization was difficult because these studies had a limited number of subjects who differed in the severity of symptoms.

SLIT is already used widely for the treatment of AR, such as that due to pollinosis, in Europe. SLIT products for general clinical application are available from Stellergenes in France and Alk Abello in Denmark. There have been objections to SLIT in the United States, probably because SCIT used only by allergists However, a Cochrane cooperative metaanalysis by Wilson showed that SLIT was effective ${ }^{22}$. In the ARIA 2000 guidelines, SLIT is recommended for AR, conjunctivitis, and asthma due to pollen or mite allergy; these guidelines also clarified that SLIT influences the natural history of allergy in the same 
way as $\mathrm{SCIT}^{23}$. The concept that the mechanism of SLIT involves IgX, which blocks all IgE, has been proposed $^{24}$. However, a reported has pointed out the problem of presentation bias in a new meta-analysis. As a result, it has been suggested that there was no significant efficacy shown in some of the studies where SLIT was reported to be effective ${ }^{25}$.

\section{SLIT Research in Japan}

Our group at Nippon Medical School began a clinical trial of SLIT for JC pollinosis in $2002^{20}$. Moreover, it is that the clinical trial of the doctor initiation was made as a high evidence in our group ${ }^{26}$ and Chiba University ${ }^{27}$, also. Our SLIT regimen involved sublingual JC allergen at a dose from 2 Japanese Allergology Units (JAU)/mL to 2,000 JAU/ $\mathrm{mL}$, with a daily increase over a 4 -week period. The maximum dose $(2,000 \mathrm{JAU} / \mathrm{mL}=20$ drops $)$ is administered twice during week 5 . From week 6 , the same dose is administered once a week through the season.

The level of JC and Japanese cypress pollen was about 12,000 grains $/ \mathrm{cm}^{2}$, which is high, during a 2005 study in Tokyo $^{26}$ Sixty patients were randomly assigned to each treatment, and a double-blind placebo-controlled examination was done. In patients with JC pollinosis, SLIT decreased the symptom score and the QOL score more than did placebo, and the improvement was by double rate compared to the placebo, especially in QOL (Fig. 3). The effect against JC pollinosis has also been studied by Chiba University and was found to be similar, with a decrease in JC-specific $\mathrm{T}$ cell clones in the peripheral blood $^{27}$. Moreover, Tr1 (which induces regulatory $\mathrm{T}$ cells) was induced by SLIT in a study by Nippon Medical School and Mie University. The induction of regulatory $\mathrm{T}$ cells is a significant effect of $\mathrm{SLIT}^{28}$.

\section{References}

1. Noon L: Prophylactic inoculation against hay fever. Lancet 1911; 1: 1572-1574.

2. Lockey RF: “ARIA”: Global guidelines and new forms of allergen immunotherapy. J Allergy Clin Immunol 2001; 108: 497-499.

3. Ito K: WHO comments and responses allergen immunotherapy: Treatment vaccine of allergosis.
Allergy 1998; 47: 749-794 (in Japanese).

4. Mirone C, et al:: Efficacy and safety of subcutaneous immunotherapy with a biologically standardized extract of Ambrosia artemisiifdia pollen in doubleblind placebo controlled study. Clin Exp Allergy 2004; 34: 1408-1414.

5. Durham SR, et al:: Long-term clinical efficacy of grass-pollen immunotherapy. N Engl J Med 1999; 341: 468-475.

6. Maestrelli P, et al.: Effect of specific immunotherapy added to pharmacologic treatment and allergen avoidance in asthmatic patients allergic to house dust mite. J Allergy Clin Immunol 2004; 111: 643-649.

7. Ameal A, et al: Double-blind and placebo-controlled study to assess efficacy snd safety of modified allergen extract of Dermatophagoides Pteronussinus in Allergic asthma. Allergy 2005; 60: 1178-1183.

8. Pifferi M, et al: Benefits of immunotherapy with a standardized Dermatophagoides pteronyssinus extract in asthmatic children: a three years prospective study. Allergy 2002; 57: 785-790.

9. Moller $\mathrm{C}$, et al: Pollen immunotherapy reduces the development of asthma in children with seasonal rhinoconjunctivitis (the PAT-study). J Allegy Clin Immunol 2002; 109: 251-256.

10. Pastorello EA, et al.: A double-blind study of hyposensitization with an alginate-conjugated extract of Dermatophagoides pteronyssinus (Conjuvac) in patients with perennial rhinitis. II. Immunological aspects. Allergy 1990; 45: 505-514.

11. Brunet C, et al.: Allergic rhinitis to ragweed pollen. I. Reassessment of the effects of immunotherapy on cellular and humoral responses. J Allergy Clin Immunol 1992; 89: 76-86.

12. McHugh SM, et al.: A placebo-controlled trial of immunotherapy with two extracts of dermatophagoides pteronyssinus in allergic rhinitis, comparing clinical outcome with changes in antigenspecific IgE, IgG, and IgG subclasses. J Allergy Clin Immunol 1990; 86: 521-531.

13. Okuda M, et al.: Effect of Anti-Allergic Treatment on Nasal Surface Basophilic Metachromatic Cells in Allergic Rhinitis. Allergy Proc 1989; 10: 23-26.

14. Kakinoki $Y$, et al.: Allergen Induced mRNA Expression of Interleukin-5, but Not of Interleukin-4 and Interferon-g, in Peripheral Blood Mononuclear Cells Obtained Before the Pollen Season Predicts the Clinical Efficacy of Immunotherapy for Seasonal Allergic Rhinitis. Scond J Immunol 2000; 51: 202-208.

15. Klimek L, et al.: Short-term preseasonal birch pollen allergoid immunotherapy influences symptoms, specific nasal provocation and cytokine levels in nasal secretions, but not peripheral T-cell responses, in patients with allergic rhinitis. Clin Exp Allergy 1999; 29: 1326-1335.

16. Bohle B, et al:: Sublingual immunotherapy induces IL-10-producing $\mathrm{T}$ regulatory cells, allergen-specific T-cell tolerance, and immune deviation. J Allergy Clin Immunol 2007; 120: 707-713.

17. Radulovic S, et al.: Grass pollen immunotherapy induces Foxp3-expressing CD4+ CD25+ cells in the nasal mucosa. J Allergy Clin Immunol 2008; 121: 1467-1472.

18. Onishi $\mathrm{M}$, et al: Side effect of specific immunotherapy in this classroom. Nose and ears 
1991; 37: 1073-1078.

19. Gotoh M, et al.: Sublingual immunotherapy for Japanese cedar pollinosis: a pilot study. Allergology International 2005; 54: 167-171.

20. Mungan D, et al.: Comparison of the efficacy of subcutaneous and sublingual immunotherapy in mite-sensitive patients with rhinitis and asthma: a placebo controlled study. Ann Allergy Asthma Immunol 1999; 82: 485-490.

21. Khinchi MS, et al.: Clinical efficacy of sublingual and subcutaneous birch pollen allergen-specific immunotherapy: a randomized, placebo controlled, double-blind, double-dummy study. Allergy 2004; 59: 45-53.

22. Wilson DR, et al:: Sublingual immunotherapy for allergic rhinitis: systematic review and meta-analysis. Allergy 2005; 60: 1-5.

23. Di Rienzo A, et al.: Long-lasting effect of sublingual immunotherapy in children with asthma due to house dust mite: a 10-years prospective study. Clin Exp Allergy 2003; 33: 206-210.

24. Durham SR, et al.: Sublingulal immunotherapy with once-daily grass allergen tablet: a randomized controlled trial in seasonal allergic rhinoconjunctivitis. J Allergy Clin Immunol 2006; 117: 802-809.

25. Nieto A, et al.: Sublingual immunotherapy for allergic respiratory diseases; an evaluation of metaanalyses. J Allergy Clin Immunol 2009; 124: 157-161.

26. Okubo K, et al.: A randomized double-blind comparative study of sublingual immunotherapy for cedar pollinosis. Allergology International 2008; 57: 265-275.

27. Horiguchi S, et al.: A randomized controlled trial of sublingual immunotherapy for Japanese cedar pollinosis. Int Arch Allergy Immunol 2008; 146: 7684.

28. Yamanaka KI, et al.: Induction of IL-10-producing regulatory $\mathrm{T}$ cells with TCR diversity by epitopespecific immunotherapy in pollinosis. J Allergy Clin Immunol 2009; 124: 842-845.

(Received, September 6, 2010)

(Accepted, October 21, 2010) 